\title{
Reducing the Number of Sutures for Vesicourethral Anastomosis in Radical Retropubic Prostatectomy
}

\author{
Evangelos M. Mazaris, Eleftherios Chatzidarellis, Ioannis M. Varkarakis, Athanasios Dellis, \\ Charalambos Deliveliotis
}

Second Department of Urology, Sismanoglio Hospital, University of Athens, Athens, Greece

\begin{abstract}
Objectives: To prospectively evaluate the outcome of using a two-suture technique for the vesicourethral anastomosis (VUA) during radical retropubic prostatectomy (RRP).

Materials and Methods: Two groups of 50 patients each underwent nerve-sparing RRP for localized prostate cancer by one surgeon. In one group, the vesicourethral anastomosis was performed using 2 Vicryl 2-0 stitches placed at the 3- and 9-o'clock positions and in the other group 6 Vicryl 2-0 stitches were placed at the 2-, 4-, 6-, 8-, 10- and 12-o'clock positions. The intraoperative and perioperative parameters analyzed were time to perform the VUA, time to remove the drain and hospitalization. The rate of incontinence, anastomotic stricture and erectile function were included in the outcome analysis.

Results: The anastomotic time differed statistically between the 2 groups (mean 3.3 minutes for the 2-suture group and 10.5 minutes for the 6-suture group, $\mathrm{p}<0.0001$ ) with similar periods of drain removal (mean 3.12 days for the 2-suture group and 3.45 days for the 6-suture group; $\mathrm{p}=0.13$ ) and hospitalization (mean 4.66 days for the 2-suture group and 5.3 days for the 6-suture group; $\mathrm{p}=0.09$ ). The functional outcome was excellent for the 2 -suture group with no patient suffering from incontinence or anastomotic strictures 1 year postoperatively, while in the 6-suture group there were 2 patients $(4 \%)$ suffering from incontinence ( 2 underwent sling procedure) and 1 patient suffered from anastomotic stricture.

Conclusion: The low number of sutures in the 2-suture VUA technique reduces operating times, does not influence perioperative and intraoperative parameters and results in excellent functional outcome.
\end{abstract}

Key words: prostatic neoplasm; prostatectomy; anastomosis, surgical; sutures; treatment outcome Int Braz J Urol. 2009; 35: 158-63

\section{INTRODUCTION}

Radical retropubic prostatectomy (RRP) is one of the treatment modalities recommended for clinically organ-confined prostate cancer. The better understanding of pelvic anatomy has led to the development of the anatomic approach of RRP (1) and to the reduction of specific complications of the operation such as incontinence, anastomotic stricture and impotence.
One of the critical steps of the operation that may influence the rate of postoperative complications is the anastomosis of the bladder to the urethral stump. Historically, the number of six sutures was described by Walsh (1) to be used for the vesicourethral anastomosis (VUA). However, this number may vary from four (2) to eight sutures, according to other experienced surgeons, with adequate functional results (3). A reduction in the number of sutures used in order to perform the VUA in radical prostatectomy 
is now becoming important in the laparoscopic era where intracorporeal suturing is difficult and intraperitoneal leak could be a problem. We examined the feasibility of an interrupted two-suture vesicourethral anastomosis technique and its effect on the outcome of prostate cancer patients after open radical retropubic prostatectomy.

\section{MATERIALS AND METHODS}

From September 2005 to October 2006, we prospectively evaluated 100 patients, divided randomly into two groups of 50 patients each, by consecutive allocation to each group, who underwent nerve-sparing RRP by a single surgeon, after obtaining institutional review board approval. Patients who had undergone preoperative androgen ablation, local radiotherapy, previous transurethral or suprapubic resection of the prostate or underwent non nerve-sparing RRP were excluded from the study.

All patients underwent bilateral pelvic lymphadenectomy and standard nerve-sparing RRP as described by Walsh (1). The bladder neck was preserved and bladder neck mucosal eversion was performed routinely using 4 to 6 circumferential absorbable sutures 3-0. In one group the vesicourethral anastomosis was performed using 2 Vicryl (Ethicon, NJ, USA) 2-0 stitches placed at the 3- and 9-o'clock positions and in the other group 6 Vicryl 2-0 stitches were placed at the 2-, 4-, 6-, 8 -, 10- and 12-o'clock positions. The VUA was carried-out on a $22 \mathrm{~F} 3$-way Couvelaire catheter, which was removed on the 10th postoperative day without performing a cystogram. Two drains were also routinely placed laterally to the anastomosis. They were removed when daily drain output was less than $50 \mathrm{~mL}$.

The following intraoperative and postoperative parameters were recorded: time to perform VUA, blood loss, pre-and postoperative hemoglobin, drain output, time to drain removal and consequent transfusions. Follow-up consisted of visits every 3 months for 1 year, during which physical examination was performed and PSA values measured. All patients were encouraged to execute pelvic floor exercises (Kegel) (4) and phosphodiesterase-5 (PDE5) inhibitors were also administered early postoperatively (5). Continence was evaluated by the number of pads used daily as reported by the patients during the follow-up visits. They were defined as continent when no more than one pad daily was required (6).

The statistical analysis was performed by Student's t-test for quantitative data and the chi-square test for categorical data. Results were considered statistically significant at $\mathrm{p} \leq 0.05$.

\section{RESULTS}

Patient characteristics such as age, PSA value, body mass index, prostate volume measured by transrectal ultrasound and Gleason score are presented in Table-1.

Table 1 - Patient characteristics.

\begin{tabular}{lccc}
\hline & 2-suture VUA & 6-suture VUA & p Value \\
\hline Mean age (range) & 64.6 years $(52-74)$ & 64.1 years $(49-76)$ & $\mathrm{p}=0.82$ \\
Mean PSA (range) & $8.44 \mathrm{ng} / \mathrm{mL}(3.5-19.58)$ & $8.59 \mathrm{ng} / \mathrm{mL}(4-28.2)$ & $\mathrm{p}=0.86$ \\
Mean Gleason score (range) & $6.19(3-9)$ & $6.15(3-8)$ & $\mathrm{p}=0.94$ \\
Mean BMI (range) & $25.7(21-38)$ & $25.9(19-40)$ & $\mathrm{p}=0.91$ \\
Mean TRUS volume (range) & $21.8 \mathrm{~cm}^{3}(16-38)$ & $22.2 \mathrm{~cm}^{3}(15-42)$ & $\mathrm{p}=0.77$ \\
\hline
\end{tabular}

$V U A=$ vesicourethral anastomosis $; B M I=$ body mass index $;$ TRUS = transrectal ultrasound. 
The analysis of the intraoperative parameters revealed a mean time to perform the vesicourethral anastomosis (VUA) of 3.3 minutes for the 2-suture technique and 10.5 minutes for the 6 -suture technique (Table-2). Urinary leakage occurred in 4 patients (8\%) in each of the two groups while the mean volume was $1125 \mathrm{~mL}$ for the 2-suture technique compared with a significantly less mean volume of leakage of $980 \mathrm{~mL}$ for the 6 -suture technique. Urinary leakage in both groups subsided spontaneously with patient mobilization and all drains in these patients had been removed by the $3 \mathrm{rd}$ or 4 th postoperative day, thus all patients were discharged without drains.

During the postoperative course the mean time to ambulation was 1 day for the 2-suture technique and 1.02 days for the 6-suture technique, the mean time to oral intake was 1.22 (range 1-4) days and 1.15 (range 1-3) days respectively. Timescale to drain removal and patient discharge did not differ statistically between the two groups (Table-2).

No significant postoperative bleeding was observed as only 2 patients (4\%) were transfused with 3 blood units in total in each group postoperatively.

Regarding the 2-suture technique, no patient suffered from incontinence 1 year postoperatively, while with the 6-suture technique, 2 (4\%) patients were incontinent in the same period and a male sling was placed successfully. No patient suffered from anastomotic stricture with the 2-suture technique while 1 patient suffered from stricture with the 6suture technique, which was endoscopically treated (Table-2).

\section{COMMENTS}

The creation of the VUA is a crucial step in RRP since it affects future outcomes and thus the quality of life of such patients postoperatively. It seems that prevention of anastomotic stricture (2) and urinary continence (7) depends on a well-healed vesicourethral anastomosis. The general principle to achieve this, regardless of the anastomotic technique used, is a watertight, tension-free anastomosis with mucosal-to-mucosal coaptation and proper urethral alignment (8).

Recently, a VUA technique using two interrupted sutures with equal outcomes while offering reduced anastomotic time was reported (6). The task of performing a 2-suture technique for the VUA is not only convenient for open RRP but may also simplify the procedure for the laparoscopic approach since suturing during laparoscopy is more challenging. Leakage was significantly higher in the 2-suture

Table 2 - Intraoperative and postoperative parameters as well as functional outcome.

\begin{tabular}{lccc}
\hline & 2-suture VUA & 6-suture VUA & p Value \\
\hline Mean time to VUA (range) & $3.32 \mathrm{~min}(2.9-4.2)$ & $10.5 \mathrm{~min}(7.5-14)$ & $\mathrm{p}<0.0001$ \\
Mean total urinary leakage (range) & $1125 \mathrm{~mL}(600-1500)$ & $980 \mathrm{~mL}(700-1310)$ & $\mathrm{p}=0.01$ \\
Mean EBL (range) & $454.17 \mathrm{~mL}(200-1000)$ & $733.63 \mathrm{~mL}(300-2500)$ & $\mathrm{p}=0.001$ \\
Mean time to ambulation & 1 day & 1.02 day & $\mathrm{p} \leq 1$ \\
Mean time to oral intake (range) & 1.22 days (1-3) & 1.15 days $(1-3)$ & $\mathrm{p}=0.33$ \\
Mean time to drain removal (range) & 3.12 days (2-5) & 3.45 days $(2-6)$ & $\mathrm{p}=0.13$ \\
Mean hospital stay (range) & 4.66 days (3-10) & 5.3 days $(3-11)$ & $\mathrm{p}=0.09$ \\
Incontinence at 12 months & 0 patients $(0 \%)$ & 2 patients $(4 \%)$ & $\mathrm{p}=0.24$ \\
Anastomotic stricture & 0 patients & 1 patient & \\
\hline
\end{tabular}

$V U A=$ vesicourethral anastomosis; $E B L=$ estimated blood loss. 
technique; however, it stopped spontaneously with ambulation and was drained effectively, thus resulting in no patients with incontinence or anastomotic stricture. Our two-suture technique for the VUA is faster and less challenging than using a four (2), six (1) or eight-suture (3) or even a running suture (9) technique since it is obvious that by using more sutures is more time-consuming and complicated. Some surgeons place their sutures in the urethra before dividing it completely and leave them on the surgical table until the bladder neck is ready for the VUA; while others place their sutures creating the VUA after the prostate has been removed. In the first case sometimes sutures become entangled increasing the surgical time. It is evident that by using only two sutures entanglement is more difficult and disentanglement easier. Furthermore, in contrast to a recent study using the two-suture technique (6) the placement of sutures in the 3 and 9 o'clock positions instead of the 6 and 12 o'clock, avoids the rectum and the rectourethralis muscle dorsally and branches of the Santorini plexus anteriorly.

Moreover, in the present series preservation of the bladder neck was selected since earlier return to continence and a reduction in the stricture rate have been reported when using such technique (10). We also everted the bladder mucosa permitting a close coaptation with the urethral mucosa and tried to avoid interposition of perivesical fat when tying the sutures (11).

In the present series no patient with the 2suture and 1 patient with the 6-suture technique developed an anastomotic stricture, which rates vary in the literature from $0.5 \%$ to $32 \%$ (12), despite having 4 patients in each group with urinary leak in the early postoperative period, which has been described as a risk factor (13) for stricture formation. The incidence of urinary leak for the 2-suture technique was higher compared with the 6-suture technique in accordance with other studies (6). In all of these patients the leak ceased spontaneously with ambulation and an unobstructed urethral catheter. On the contrary, it seems that the degree of tightness of the anastomosis (9) with a compromised vascular supply to the bladder neck and the urethra is a predisposing factor for stricture formation, thus by using two sutures we avoid such tightness. Furthermore, we used a 3-way wide catheter of $22 \mathrm{~F}$ without traction, in order to achieve healing of the suture line since it has been proven that the incidence of anastomotic stricture is reduced with a wider caliber of the anastomosis (14). A large bore catheter results in better drainage, which decreases the potential for leakage. Postoperative bleeding was also minimal in our patients, thus avoiding another risk factor for stricture formation. By using drains, especially large ones in the early postoperative period, better removal of fluid or blood from the anastomotic site is achieved, in order to avoid fibrosis and stricture formation.

The rates of incontinence after RRP have been reported from $2.5 \%$ to $87 \%$ (15), yet a 12 -month period is necessary before defining a patient's continence status (16). Several risk factors have been reported to contribute to continence after RRP such as patient age, disease stage, surgical technique, preoperative continence and previous transurethral resection of the prostate (17). In our series the rate of incontinence was very low, consistent with other reports (9) and it is noteworthy that no patient suffered from incontinence 1 year after the operation with the 2 -suture technique while 2 patients were incontinent with the 6-suture technique.

In order to preserve continence, the membranous urethra, the sphincter mechanism and its innervations as well as the anastomotic blood supply should be preserved. The "continence nerves" seem to be damaged during blunt dissection of the posterior periurethral tissues near the junction of the levator ani muscle and during placement of the anastomotic sutures at the 5- and 7-o'clock positions (6). By placing sutures in the 3- and 9-o'clock positions we can avoid such nerves. Additionally, by using the nerve-sparing procedure we improved our continence rates (18), which are possibly attributed to the meticulous dissection of the nerves from the apex of the prostate instead of the preservation of the neurovascular bundle. Furthermore, preservation of the bladder neck especially of its circular fibers, as in this series of patients, contributes to return of continence (19).

In this initial number of patients we removed the catheter after 10 days although being aware of the accumulating reports of early catheter removal (20). Nevertheless, we must be aware that there are 
differences in the surgical technique and the number of sutures used for the VUA. Prospective comparative studies with several techniques for VUA are required, in order to confirm the superior results of a 2-suture technique. However, we must acknowledge that these 50 patients, with this specific surgical technique of reduced number of sutures for the VUA, had excellent outcome with minimal complications compared with the same number of patients with a 6-suture technique, thus, proving effective, less challenging and convenient for use in open RRP. We must also acknowledge that in our study patients were discharged in their majority between the 4th and 5th postoperative day, due to special circumstances existing in our country (long distance from permanent residence, National Health System environment, no pressure by insurance companies, etc).

\section{CONCLUSION}

The reduced number of sutures used for the VUA in the present study reduced surgical time safely, is easier to perform and achieved excellent functional outcomes. Although urinary extravasation was higher in the intraoperative period, it was managed conservatively and stopped spontaneously having no effect on stricture formation. The reduced number of sutures for the VUA seems to have a lower incidence of incontinence and anastomotic stricture resulting in minimal late complications after RRP.

\section{CONFLICT OF INTEREST}

None declared.

\section{REFERENCES}

1. Walsh PC: Anatomic radical prostatectomy: evolution of the surgical technique. J Urol. 1998; 160: 241824.

2. Borboroglu PG, Sands JP, Roberts JL, Amling CL: Risk factors for vesicourethral anastomotic stricture after radical prostatectomy. Urology. 2000; 56: 96100 .
3. Srougi M, Nesrallah LJ, Kauffmann JR, Nesrallah A, Leite KR: Urinary continence and pathological outcome after bladder neck preservation during radical retropubic prostatectomy: a randomized prospective trial. J Urol. 2001; 165: 815-8.

4. Van Kampen M, De Weerdt W, Van Poppel H, De Ridder D, Feys H, Baert L: Effect of pelvic-floor reeducation on duration and degree of incontinence after radical prostatectomy: a randomized controlled trial. Lancet. 2000; 355: 98-102.

5. Montorsi F, Briganti A, Salonia A, Rigatti P, Burnett $\mathrm{AL}$ : Current and future strategies for preventing and managing erectile dysfunction following radical prostatectomy. Eur Urol. 2004; 45: 123-33.

6. Gallo L, Perdonà S, Autorino R, Menna L, Claudio L, Marra L, et al.: Vesicourethral anastomosis during radical retropubic prostatectomy: does the number of sutures matter? Urology. 2007; 69: 547-51.

7. Steiner MS, Morton RA, Walsh PC: Impact of anatomical radical prostatectomy on urinary continence. J Urol. 1991; 145: 512-4; discussion 514-5.

8. Walsh PC, Quinlan DM, Morton RA, Steiner MS: Radical retropubic prostatectomy. Improved anastomosis and urinary continence. Urol Clin North Am. 1990; 17: 679-84.

9. Miki T, Okihara K, Ukimura O, Usijima S, Yoneda K, Mizutani Y, et al.: Running suture for vesicourethral anastomosis in minilaparotomy radical retropubic prostatectomy. Urology. 2006; 67: 410-2.

10. Shelfo SW, Obek C, Soloway MS: Update on bladder neck preservation during radical retropubic prostatectomy: impact on pathologic outcome, anastomotic strictures, and continence. Urology. 1998; 51: 73-8.

11. Tomschi W, Suster G, Höltl W: Bladder neck strictures after radical retropubic prostatectomy: still an unsolved problem. Br J Urol. 1998; 81: 823-6.

12. Popken G, Sommerkamp H, Schultze-Seemann W, Wetterauer U, Katzenwadel A: Anastomotic stricture after radical prostatectomy. Incidence, findings and treatment. Eur Urol. 1998; 33: 382-6.

13. Surya BV, Provet J, Johanson KE, Brown J: Anastomotic strictures following radical prostatectomy: risk factors and management. J Urol. 1990; 143: 755-8.

14. McCarthy J, Catalona W: Nerve Sparing Radical Retropubic Prostatectomy. In: Marshall F (ed.), Textbook of Operative Urology, 1st ed., Philadelphia, WB Saunders. 1996, pp. 537-44.

15. Lepor H, Kaci L, Xue X: Continence following radical retropubic prostatectomy using self-reporting instruments. J Urol. 2004; 171: 1212-5. 


\section{Vesicourethral Anastomosis in RRP}

16. Poore RE, McCullough DL, Jarow JP: Puboprostatic ligament sparing improves urinary continence after radical retropubic prostatectomy. Urology. 1998; 51: 67-72.

17. Eastham JA, Kattan MW, Rogers E, Goad JR, Ohori $\mathrm{M}$, Boone TB, et al.: Risk factors for urinary incontinence after radical prostatectomy. J Urol. 1996; 156: 1707-13.

18. Burkhard FC, Kessler TM, Fleischmann A, Thalmann GN, Schumacher M, Studer UE: Nerve sparing open radical retropubic prostatectomy--does it have an impact on urinary continence? J Urol. 2006; 176: 18995.

19. Selli C, De Antoni P, Moro U, Macchiarella A, Giannarini G, Crisci A: Role of bladder neck preservation in urinary continence following radical retropubic prostatectomy. Scand J Urol Nephrol. 2004; 38: 32-7.

20. Patel R, Lepor H: Removal of urinary catheter on postoperative day 3 or 4 after radical retropubic prostatectomy. Urology. 2003; 61: 156-60.

\section{Correspondence address:}

Dr. Evangelos Mazaris

89 Agiou Ioannou Street

Agia Paraskevi, Athens, 15342, Greece

Fax: + $30210804-4703$

E-mail: evmazaris@yahoo.gr 